\title{
EVALUATING THE EFFECTIVENESS OF THE CORE CONTENT OF THE INCREDIBLE YEARS WITH AND WITHOUT VISUAL PERFORMANCE FEEDBACK FOR PARENTS OF CHILDREN WITH AUTISM
}

\author{
Tia R. Schultz \\ Dr. Janine Stichter, Dissertation Supervisor
}

\begin{abstract}
Many children with autism display externalizing behavior due to challenges they face in the areas of communication, social skills and repetitive behavior or restricted interests. Parents have reported that it is difficult to know how to address these behaviors. Parent training programs have been found to increase parents' skills and decrease child externalizing behavior. The purpose of this study was to evaluate the effectiveness of the core content of The Incredible Years parent training program with and without Visual Performance Feedback in: a) increasing parent skills and b) decreasing child externalizing behavior. The secondary purpose was to assess the extent to which parents found the interventions socially valid.

Using a multiple baseline design across parent-child dyads, data were collected on parent behaviors (use of clear commands, praise and descriptive play statements) and child behaviors (compliance, prompted compliance, on-task/appropriate behavior and externalizing behaviors). Phases included baseline, parent training and feedback.

Results indicated that when the parent variables of clear commands, praise and descriptive play statements were combined, all parents made mild increases in their use of those skills during each phase. However, there was variability in specific variables across parents and phases. On the Eyberg Child Behavior Inventory, all parents reported decreases in child externalizing behavior from pre to post intervention. However, there was variability in specific variables across children and phases. Interpretation of these results is provided along with study limitations and future directions for research.
\end{abstract}

